Résumés des conférences et travaux

\title{
Littérature philosophique à Byzance et sa postérité à l'époque moderne
}

\section{Michel Cacouros}

\section{(2) OpenEdition}

\section{Journals}

Édition électronique

URL : https://journals.openedition.org/ashp/659

DOI : 10.4000/ashp.659

ISSN : 1969-6310

\section{Éditeur}

Publications de l'École Pratique des Hautes Études

\section{Édition imprimée}

Date de publication : 1 octobre 2009

Pagination : 92-101

ISSN : 0766-0677

\section{Référence électronique}

Michel Cacouros, «Littérature philosophique à Byzance et sa postérité à l'époque moderne », Annuaire de l'École pratique des hautes études (EPHE), Section des sciences historiques et philologiques [En ligne], 140 | 2009, mis en ligne le 08 octobre 2009, consulté le 06 juillet 2021. URL : http:// journals.openedition.org/ashp/659; DOI : https://doi.org/10.4000/ashp.659 


\title{
LITTÉRATURE PHILOSOPHIQUE À BYZANCE ET SA POSTÉRITÉ À L'ÉPOQUE MODERNE
}

\author{
Maître de conférences : M. Michel Cacouros
}

Programme de l'année 2007-2008 : I. Philosophie byzantine et post-byzantine. L'exégèse d'Aristote : naissance et développement de l'écrit philosophique à caractère interprétatif (synopses, paraphrases, commentaires). - II. Histoire de la culture et de l'enseignement à Byzance et dans l'après-Byzance : les disciplines du Trivium et du Quadrivium (Arts libéraux) : corpus et manuels (suite); terminologie et lexique de l'enseignement.

De même que les dernières années, la première partie du séminaire a porté sur l'histoire de la philosophie byzantine et post-byzantine, plus précisément sur l'exégèse d'Aristote à Byzance et dans l'après-Byzance, alors que la seconde a été consacrée à la culture et l'enseignement, et, notamment, à la tradition du Trivium et du Quadrivium (Arts libéraux) à Byzance et dans l'après-Byzance.

\section{Naissance et évolution du commentaire aristotélicien à Byzance : des prôto-commentaires aux commentaires de Michel d'Éphèse, d'Eustrate de Nicée et de Théodore Prodrome}

L'écrit philosophique à caractère exégétique a revêtu à Byzance plusieurs formes. Dans le cadre de notre séminaire de 2006-2007 ${ }^{1}$, nous avions présenté un aperçu sur les formes que nous désignons, depuis quelques années déjà ${ }^{2}$, comme « latérales » afin de les différencier de celle du commentaire; durant cette même année, nous avions également attiré l'attention sur le fait que le commentaire constituait la forme la plus achevée de l'écrit exégétique, notamment du point de vue de la continuité de l'acte exégétique, et, en même temps, celle qui avait connu l'évolution la plus significative à Byzance; toutefois, il ne correspondait pas, en tout cas dans l'état actuel de nos connaissances, à la forme la plus ancienne de l'écrit exégétique à Byzance. Ainsi, le terme utilisé de « forme latérale » ne visait pas à établir un rapport chronologique quelconque entre les «formes latérales » et celle du commentaire, mais, surtout, à mettre l'accent sur le fait que l'évolution des premières a été moins marquée que celle du commentaire, et que, à ce titre, ce dernier constituait la forme la plus achevée de l'écrit exégétique.

C'est pour cette raison que, pour 2007-2008, nous avions jugé nécessaire de donner un aperçu sur la naissance et l'évolution du commentaire à Byzance. Cette décision

1. Voir notre rapport dans Annuaire. Résumés des conférences et travaux de l'École pratique des hautes études, Section des sciences historiques et philologiques, 139ª année (2006-2007), p. 55-63 et, en particulier, p. 55.

2. Voir les références fournies ibidem, p. 55 n. 1. 
s'imposait également pour la raison suivante : si l'identification, les caractéristiques et l'histoire des «formes latérales » à Byzance n'avaient pas été étudiées avant que nous nous y consacrions, la même remarque était de mise pour le commentaire à Byzance. En effet, sa naissance et son évolution n'avaient pas été passées en revue, pas plus que sa composition (la situation était la même pour la structure originelle du commentaire aussi bien que pour sa structure évoluée) ou les termes qui avaient servi à le désigner. La raison en était que les approches qui, jusqu'à présent, avaient été consacrées à la philosophie byzantine visaient surtout à établir la biographie des grands philosophes byzantins et à enregistrer les œuvres qu'ils avaient rédigées et, à ce titre, elles n'étaient pas destinées à offrir une approche plus globale et synthétique sur l'histoire de l'écrit exégétique de contenu philosophique.

C'est dire que l'exposé effectué dans notre séminaire visait à combler une véritable lacune; si le résumé qui suit en donne une idée extrêmement sommaire, signalons qu'une présentation riche et détaillée figure dans l'étude plus générale, actuellement en voie d'achèvement, sur l'écrit exégétique de contenu philosophique à Byzance ${ }^{1}$; quant aux éléments qui, dans la suite, porteront sur l'activité de Michel d'Éphèse, ils ont été empruntés à la monographie que nous consacrons actuellement à cet érudit et à son œuvre ${ }^{2}$. Pour ces raisons, parmi les divers aspects qui ont été passés en revue dans notre séminaire de l'année 2007-2008, nous nous contenterons de donner, dans la suite, uniquement quelques éléments sur la naissance du commentaire et, surtout, sur les premières étapes de son évolution $\left(\mathrm{X}^{\mathrm{e}}-\mathrm{XII}^{\mathrm{e}}\right.$ siècle); pour des raisons de concision, certains apports, comme celui de Michel Psellos, seront entièrement passés sous silence.

a. Aux prémisses du commentaire byzantin. - Même si le commentaire se caractérise et se définit, de manière générale, par la continuité de l'acte exégétique, il faut préciser que plusieurs types de commentaire ont été pratiqués à Byzance, correspondant à des conceptions variées de cet écrit exégétique et fortement marquées par l'évolution que celui-ci a connue. Il semblerait que le commentaire à Byzance se présentait à l'origine comme un agglomérat composé d' « unités exégétiques » (autrement dit d'éléments exégétiques autonomes, qui correspondaient surtout à des développements de nature exégétique ou autre, à des lieux communs, ou à des exemples ${ }^{3}$ ) couvrant la totalité (ou, en tout cas, la majeure partie) de l'œuvre aristotélicienne commentée. Ces unités étaient juxtaposées; faute de transition, le passage de l'une à l'autre devait se faire grâce à l'incipit du passage aristotélicien scommenté, donné en tête de l'unité exégétique concernée ; ainsi, les incipit servaient pour ainsi dire de tampons, permettant de séparer les unités exégétiques successives. Ces dernières étaient pour la plupart

1. Cet ouvrage, auquel nous nous consacrons depuis plusieurs années, est annoncé, entre autres, ibidem, p. $55 \mathrm{n} .1$.

2. Ce travail était déjà annoncé dans notre contribution : «Le commentaire aristotélicien et ses aspects peu connus : caractéristiques, tendances et perspectives », Hypomnèma stè philosophia, 4 (2006), p. 155-190 et, en particulier, p. 169.

3. Sur les éléments pouvant constituer les « unités exégétiques » de l'écrit exégétique, voir notre rapport dans Livret-Annuaire de l'École pratique des hautes études, Section des sciences historiques et philologiques, $135^{\mathrm{e}}$ année, n. s. 20 (2004-2005), p. 103-110 et, en particulier, p. 105-106. 
empruntées à des corpus de scholies ou, de manière plus générale, à l'ensemble des éléments exégétiques qui étaient en circulation pendant les périodes envisagées et que nous avons jadis désigné par le terme d' « amas exégétique $»^{1}$.

Ces commentaires constituaient des ensembles relativement hétérogènes, tant du point de vue de la forme (ainsi qu'il vient d'être signalé, les transitions d'un passage à l'autre n'étaient pas marquées) que du contenu, étant donné la variété du matériel réuni dans leur confection : il en résultait que les développements dus aux premiers exégètes byzantins étaient souvent parmi les rares éléments textuels qui n'avaient pas été empruntés à l'interprétation néoplatonicienne ou aux premiers corpus de scholies confectionnés à Byzance. L'uniformité des positions interprétatives exprimées dans les différents passages choisis (qui constituaient souvent la réprise littérale du modèle utilisé) ne constituait pas une règle, voire même une nécessité, aux yeux des exégètes, qui ressemblaient davantage à des excerptores qu'à de véritables auteurs. Étant donné les raisons évoquées, ces « commentaires » devaient ressembler plus à des corpus de scholies qu'à de véritables commentaires ${ }^{2}$. Afin de mettre en relief le caractère souvent hétéroclite de ce type d'écrit, relativement rudimentaire, nous l'avons désigné comme « commentaire à scholies » ou comme « prôto-commentaire ».

b. Michel d'Éphèse face au " commentaire à scholies » : respecter la tradition tout en la modifiant. - À la fin du XI ${ }^{\mathrm{e}}$-début du XII ${ }^{\mathrm{e}}$ siècle, Michel d'Éphèse pratiquait encore ce type de commentaire. Toutefois, comme no le montrons dans la monographie que nous lui consacrons actuellement, il a su apporter un certain nombre de modifications qui ont été capitales pour l'évolution ultérieure du commentaire à Byzance.

Toutefois, afin de saisir l'importance des innovations pratiquées par Michel d'Éphèse et pour pouvoir en rendre compte, il fallait tout d'abord résoudre un problème (le premier problème concernant cet exégète), particulièrement épineux : la tradition manuscrite de son œuvre est, à notre avis, fragmentaire, lacunaire et incomplète. Signalons que la partie la plus considérable de son œuvre (correspondant à plusieurs commentaires) a été éditée dans les Commentaria in Aristotelem graeca, vaste entreprise éditoriale qui a été réalisée sous les auspices de l'Académie prussienne de Berlin $^{3}$. Or, cette édition des textes éphésiens présente, suivant les comptes rendus qui en ont été faits ${ }^{4}$ et, aussi, d'après nous, plusieurs problèmes : ils portent surtout sur le fait que, parfois, les éditeurs ont passé sous silence les problèmes d'attribution et les questions liées à l'établissement des textes éphésiens édités; de plus, ils n'ont pas su expliquer les raisons pour lesquelles certains textes éphésiens ont parfois été transmis sous forme de différentes recensions.

1. Voir ibidem, p. 104-106.

2. Sur les rapports entre les scholies et les commentaires - en attendant la parution de notre ouvrage sur l'aristotélisme à Byzance -, l'on peut consulter notre contribution : « Le commentaire aristotélicien et ses aspects peu connus : caractéristiques, tendances et perspectives », p. 167-168.

3. Voir la liste des publications fournie ibidem, p. 169 n. 25.

4. Les recensions de K. Prächter, comprenant plusieurs critiques et réserves à l'égard des éditions éphésiennes, en font partie; voir la note suivante. 
En somme, l'œuvre éphésienne pose tout d'abord un problème de reconstitution et K. Prächter ${ }^{1}$ a été le premier à montrer que cette œuvre était plus étendue qu'on ne le pensait à l'époque pour ce qui concerne le nombre des œuvres aristotéliciennes commentées. Depuis, d'autres travaux sont venus s'ajouter à ceux de K. Prächter (R. Browning, A. Preuss, D. Harlfinger, L. Benakis...); nous les avons repris et enrichis dans le cadre de notre étude et espérons que, à présent, nous avons pu reconstituer - dans la mesure de nos moyens - la majeure partie de son œuvre. Ainsi, c'était seulement au terme de ce travail de reconstitution qu'il a été possible d'estimer à sa juste valeur l'apport exégétique de Michel d'Éphèse; il en sera très brièvement question dans la suite.

En effet, en premier lieu, il a « amalgamé » les lieux communs exégétiques habituellement utilisés d'une manière qui ne correspondait pas à celle qui était en cours à son époque. Il a essayé de remédier au manque de transitions dans l'écrit exégétique en développant certains types d'articulations et, aussi, en introduisant des remarques d'ordre personnel. Les renvois que Michel d'Éphèse effectuait, lors de la rédaction d'un ouvrage, aux autres exégèses composées par lui, permettaient, eux aussi, de donner une forme plus complète au « commentaire à scholies ». En deuxième lieu, parmi les éléments provenant de l' «amas exégétique » mentionné plus haut, il a su sélectionner et développer ceux qui avaient peu servi jusqu'à son époque; il a également su localiser des sources antiques entièrement inexplorées, autrement dit qui n'avaient pas été reprises, de manière partielle ou entière, dans cet « amas exégétique ». En troisième lieu, il a su faire preuve d'originalité, car il a écrit et composé lui-même des unités exégétiques qui étaient tout à fait similaires à celles, en principe d'origine antique, qui figuraient dans l' " amas exégétique »; la similitude même était parfois si marquée que l'on a pendant longtemps cru qu'il s'agissait de commentaires anciens. En somme, tout en imitant l'exégèse déjà existante (autrement dit tout en suivant l'idéal de la mimèsis que les Byzantins avaient appliqué à l'égard de la littérature grecque antique), Michel d'Éphèse a fourni une œuvre originale, dans laquelle font parfois jour des réflexions personnelles qui semblent même très modernes ${ }^{2}$.

Dernier point : Michel d'Éphèse a enrichi de manière considérable l'exégèse purement byzantine, car, en fait, il est le premier Byzantin à avoir fourni un corpus complet de commentaires byzantins destiné à couvrir l'ensemble de l'œuvre aristotélicienne.

1. Notamment dans : K. Prächter, « Michaelis Ephesii in libros de partibus animalium de animalium motione de animalium incessu commentaria. Consilio et auctoritate academiae litterarum regiae Borussicae edidit Michael Hayduck (Comment. in Arist. Graeca, ed. cons. et auct. acad. litt. reg. Boruss. vol. XXII pars II). Berlin. G. Reimer, 1904, XIV, 193 S. », Göttingische Gelehrte Anzeigen der Königl. Gesellschaft der Wissenschaften, 168, VII (1906), p. 861-907; Idem, « Abteilung: Die griechischen Aristoteleskommentare, Commentaria in Aristotelem Graeca edita consilio et auctoritate academiae litterarum regiae Borussicae. Berolini, typis et impressis G. Reimeri », Byzantinische Zeitschrift, 18 (1909), p. 516-538 (cette recension a été traduite en anglais par V. Caston, sous le titre : « Review of the Commentaria in Aristotelem graeca », dans R. Sorabji (éd.), Aristotle Transformed. The Ancient Commentators and Their Influence, Londres, 1990, p. 31-54).

2. Cet aspect a été souligné pour la première fois par O. Immisch (éd.), Aristotelis Politica, post Fr. Susemihlium recognouit... Editio altera correctior, Leipzig, ${ }^{1} 1909$ (Bibliotheca Teubneriana), p. XI sq., repris dans la deuxième édition (1929), p. XII $s q$. Depuis, il a souvent été évoqué dans la bibliographie éphésienne. 
En un mot, il est, à notre avis (cet avis étant exprimé et publié pour la première fois dans ce contexte), le premier auteur byzantin qui a su s'émanciper de la tradition exégétique antique, en particulier de la tradition néoplatonicienne, pour créer le premier corpus exégétique entièrement byzantin destiné à couvrir l'ensemble - ou presque du Corpus aristotelicum.

Ainsi, l'avis, souvent exprimé jusqu'à présent, que Michel d'Éphèse a été le premier commentateur à interpréter les traités biologiques d'Aristote, qui n'avaient pas été commentés (ou qui ne l'avaient été qu'à titre exceptionnel) depuis l'Antiquité jusqu'à son époque, est certes vrai, mais il demande à être complété et élargi, voire même modifié, en fonction de notre affirmation. En effet, le fait que la recherche se soit attardée sur ces commentaires est certes compréhensible (d'autant plus qu'il est lié à la question de la transmission de l'œuvre éphésienne), mais il risque d'altérer la véritable image de son corpus exégétique, que nous avons désigné sous le titre de Corpus ephesianum.

Suivant l'avis exprimé pour la première fois par R. Browning, Michel d'Éphèse a exercé son activité de commentateur sur la demande de la princesse porphyrogénnète Anne Comnène (2 décembre 1083-ca 1153), notamment lorsque celle-ci s'est retirée au monastère de la Théotokos Kécharitôménè, suite au décès de son père Alexis $\mathrm{I}^{\mathrm{er}}$ Comnène en 1118 et au coup d'État avorté contre son frère, le futur Jean II (13 septembre 1087-8 avril 1143) ${ }^{1}$. Cette position de R. Browning reposait sur l'interprétation d'un passage figurant dans l'oraison funèbre d'Anne Comnène composée dans les années 1153-1155 par l'hypomnèmatographe Georges Tornikès ${ }^{2}$. Sans entrer dans les détails d'une démonstration qui n'a pas de place dans ce contexte, contentons-nous de signaler que, d'après nous, cette position, qui a reçu l'accord de plusieurs chercheurs, devra être modifiée en partie : en effet, l'activité exégétique de Michel d'Éphèse est sans doute due à l'instigation d'Anne Comnène, mais, à notre avis, elle était très largement avancée et presque achevée lors la retraite forcée de cette dernière en 1118 ; et c'est pour rédiger les commentaires qui faisaient encore défaut au Corpus ephesianum, mais, aussi, afin de remplacer certains commentaires éphésiens qui avaient été conçus et réalisés dans une (trop) stricte application du concept de « commentaire à

1. R. Browning, «An unpublished funeral oration on Anna Comnena », Proceedings of the Cambridge Philological Society, 188, n. s. 8 (1962), p. 1-12, en particulier p. 6-8 (repris dans Idem, Studies on Byzantine History, Literature and Education, Londres 1977 [Variorum Reprints CS, 59], étude $\mathrm{n}^{\circ} 7$ (même pagination), et dans Aristotle Transformed, étude no 17, p. 393-406, et, en particulier p. 399401). Cette hypothèse a été reprise, entre autres, par A. C. Lloyd, « The Aristotelianism of Eustratios of Nicaea », dans J. Wiesner (éd.), Aristotelis Werk und Wirkung, ouvrage dédié à P. Moraux, vol. II, Berlin - New York 1987, p. 341-351, et, en particulier, p. 341-342. Voir aussi Lettres et discours de Georges et Dèmètrios Tornikès, introduction, texte, analyse, traduction et notes par J. Darrouzès, Paris, 1970 (Le monde byzantin), p. 20-24.

2. Quatre extraits de ce discours funèbre ont été édités par R. Browning, «An unpublished funeral oration on Anna Comnena », p. 11-12 (repris dans Idem, Studies on Byzantine History, Literature and Education, et dans Aristotle Transformed : les extraits en grec n'y ont pas été reproduits, mais ils ont été remplacés, p. 404-405, par une traduction en anglais). L'oraison funèbre a été éditée et traduite, dans sa totalité, par J. Darrouzès dans Lettres et discours de Georges et Dèmètrios Tornikès, p. 220-323 (le passage en question figure à la p. 283, 1. 4-7). 
scholies » qu'Anne Comnène avait, à notre avis, fait appel à Eustrate de Nicée, dont il sera question dans la suite.

En effet, Michel d'Éphèse a adopté, comme il a été signalé plus haut, le type de commentaire se présentant comme un recueil de scholies, en règle générale désigné sous le nom de scholai, et qui était très probablement (encore) en usage à son époque. Or, cette conception de l'écrit exégétique semble être dépassée vers la fin de la vie de Michel; plus précisément, elle semble avoir évolué vers une conception du commentaire reposant sur un meilleur agencement des éléments qui le composaient, avec des articulations et des transitions qui étaient plus systématiques et mieux marquées.

En somme, l'œuvre de Michel d'Éphèse était particulièrement considérable du point de vue de l'étendue, de la forme et du contenu exégétiques. De toute évidence, elle était destinée à une large diffusion à Byzance, mais elle a subi, d'après l'étude que nous lui avons consacrée, deux mésaventures majeures, qui sont à l'origine de sa « disparition » partielle. Ces accidents sont à l'origine de l'état souvent fragmentaire de sa transmission mais, également, des fausses attributions qui en ont été faites.

La première mésaventure résultait du fait que, à peine réalisée ou, plus précisément, avant même d'être entièrement achevée, cette œuvre a été dépassée, notamment du point de vue de la forme exégétique choisie, par celle qu'a adoptée Eustrate de Nicée : cette dernière, plus riche, mieux structurée, et plus « moderne » (en tout cas, aux yeux d'Anne Comnène, qui avait demandé le concours d'Eustrate), semble avoir porté ombrage sur une partie considérable de l'œuvre de Michel. Toutefois, étant donné que certaines exégèses éphésiennes étaient uniques à Byzance, et que, de plus, cette œuvre avait cet aspect si « antique », ce problème aurait sans doute été dépassé.

La seconde mésaventure qu'a connue l'œuvre éphésienne est que, diffusée pendant moins d'un siècle, elle a subi les conséquences de la prise de Constantinople en 1204 par les Latins de la quatrième croisade. Ainsi, alors que, parmi les exégèses éphésiennes, plusieurs étaient largement utilisées avant 1204, après cette date, elles semblent avoir cessé de circuler, ou, pire, elles ont probablement été « dépecées » en extraits, conformément à l'effort de sauvegarde déployé par les Byzantins à Nicée ${ }^{1}$. De plus,

1. Le procédé de « dépeçage » de textes sous forme d'extraits était largement employé à Byzance : en témoigne le fait qu'il était sans cesse pratiqué dans le cadre de la confection des florilèges, des encyclopédies...; toutefois, son usage semble avoir été amplifié à l'empire de Nicée (1204-1261) par les Byzantins soucieux de sauvegarder leur patrimoine culturel menacé; sur les différents aspects que revêtait le « dépeçage » et sur son intensification durant cette période, voir notre contribution : « La philosophie et les sciences du Trivium et du Quadrivium à Byzance de 1204 à 1453 entre tradition et innovation : les textes et l'enseignement, le cas de l'école du Prodrome (Pétra) », dans M. Cacouros et M.-H. Congourdeau (éd.), Philosophie et Sciences à Byzance de 1204 à 1453. Les textes, les doctrines et leur transmission. Actes de la table ronde organisée au XX $X^{e}$ Congrès international d'études byzantines (Paris, 2001), introduction de † Jean Irigoin, Peeters, Louvain - Paris - Dudley, 2006 (Orientalia Lovaniensia Analecta,146), p. 1-51, et, en particulier, p. 34-36. Sur la manière dont les Byzantins confectionnaient les « ouvrages à citations », voir notre contribution : «L'enseignement des disciplines littéraires (Trivium), mathématiques (Quadrivium) et de la philosophie à Byzance : conception et organisation, pratiques de l'oral et techniques de l'écrit », dans De l'Antiquité à nos jours : histoire et méthodes de l'enseignement, actes du colloque historique international organisé par M.-O. Munier, abbaye-école de Sorèze, 26-27 octobre 2006, publié aux Presses du centre universitaire Champollion, Albi, 2007, p. 1-83 ( $2^{\text {nd }}$ volume des actes, avec pagination indépendante du premier) et, en particulier, p. 70-79 (avec un dessin schématisant les travaux de « sélection » effectués par les Byzantins). 
étant donné la similitude «mimétique » des exégèses de Michel avec les commentaires anciens, son nom a souvent disparu au profit de noms antiques plus illustres. Ainsi, d'une part, certaines œuvres de Michel d'Éphèse ont été transmises, dans la tradition manuscrite, sous des fausses attributions, d'autre part, certaines autres ont été conservées uniquement sous forme d'extraits au sein de recueils scholiastiques.

Ces problèmes n'ont pas été résolus dans l'édition des commentaires éphésiens effectuée dans le cadre de l'entreprise éditoriale des Commentaria in Aristotelem graeca. Bien au contraire, la présentation des commentaires éphésiens au sein de cette édition est susceptible d'induire le lecteur en erreur : ainsi, les éditeurs ont parfois réuni des extraits éphésiens provenant de différents corpus scholiastiques, sans toutefois attirer l'attention sur le caractère factice des choix textuels effectués et de la présentation adoptée (par exemple, pour les incipit aristotéliciens figurant en tête des passages) ${ }^{1}$ dans leur édition ; ils n'ont pas su non plus expliquer, comme il a été signalé précédemment, les raisons pour lesquelles certaines exégèses éphésiennes existaient sous forme de plusieurs recensions : en effet, ces dernières correspondaient très probablement à des « éditions » paléologues.

c. De Michel d'Éphèse à Eustrate de Nicée. — Après avoir étudié le rôle de Michel d'Éphèse dans l'évolution du commentaire à Byzance, nous nous sommes penché sur celui d'Eustrate de Nicée, métropolite de Nicée (ca 1050-ca 1120)². En effet, les premiers véritables commentaires - en tout cas au sens où nous entendons ce terme aujourd'hui - sont ceux que cet érudit a composés. Il a commenté d'une part les Seconds Analytiques, livre II, d'autre part les livres I et VI de l'Éthique à Nico$m_{\text {aque }}$. Comme il a été signalé par R. Browning (voir supra), ces commentaires ont été composés à la demande d'Anne Comnène; leur rédaction est probablement, ainsi que l'a soutenu le même érudit, postérieure à la mort de l'empereur Alexis I ${ }^{\text {er }}$ en 1118 , et remonte sans doute à l'époque où l'auteur appartenait au cycle d'intellectuels qui se réunissaient autour de la princesse porphyrogénète ${ }^{4}$. S'il en est ainsi, la rédaction de ces commentaires a peut-être eu lieu, comme l'a pensé R. Browning, après la condamnation canonique d'Eustrate en 1117 et avant sa mort, probablement survenue vers 1120 .

1. Par ailleurs, ce problème, y compris celui des incipit aristotéliciens, est d'ordre plus général et concerne plusieurs éditions de celles qui figurent dans les Commentaria in Aristotelem graeca; voir, à cet égard, nos remarques dans Livret-Annuaire de l'École pratique des hautes études, Section des sciences historiques et philologiques, 134 e année, n. s. 16 (2000-2001), p. 382-387, en particulier p. 382-383. Voir aussi Idem, «Survie culturelle et rémanence textuelle du néoplatonisme à Byzance : Éléments généraux - éléments portant sur la Logique », dans Actes du colloque international "The Libraries of the Neoplatonists" [Strasbourg, 12-14 mars 2004], Leyde - Boston - Cologne, Brill 2007 (Philosophia Antiqua, 107) p. 177-210 et, en particulier, p. 1925-197.

2. Dernière biographie de cet érudit, faite par nos soins : «Eustratios de Nicée (ca 1050-ca 1120) », dans R. Goulet (dir.), Dictionnaire des philosophes antiques, t. III (d'Eccélos à Juvénal), CNRS Éditions, Paris, 2000, notice $n^{\circ} 163$, p. 378-388 (avec bibliographie antérieure).

3. Ces commentaires ont été édités dans la colletion Commentaria in Aristotelem graeca (volumes XXI, 1 ; XX ; XXII, 3 : voir les références fournies dans la notice consacrée à Eustrate). Une nouvelle édition du commentaire eustratien aux Seconds Analytiques, livre II, est en préparation par nos soins.

4. Voir supra. 
Toutefois, ainsi que nous l'avons soutenu plus haut et tout en modifiant l'hypothèse formulée à cet égard par R. Browning, les exégèses qu'Eustrate a consacrées aux livres I et VI de l'Éthique à Nicomaque doivent dater de la même époque que les dernières exégèses composées par Michel d'Éphèse, ou leur sont de peu postérieures. En effet, si l'on admet qu'Eustrate et Michel ont collaboré dans le cadre de l'exégèse de cet ouvrage d'Aristote, les deux ensembles (d'une part, les exégèses eustratiennes aux livres I et VI, d'autre part, les exégèses éphésiennes aux livres V, IX et X) sont contemporains; si, au contraire, Michel d'Éphèse a tout d'abord commenté les livres $\mathrm{V}$, IX et X, puis, étant donné qu'il ne pouvait plus effectuer la tâche qui lui restait à réaliser (soit à cause de son âge avancé, soit parce qu'il venait de décéder) et qu'Eustrate a alors été appelé par Anne Comnène afin qu'il complète cette œuvre, qui risquait de rester inachevée, dans ce cas, l'exégèse d'Eustrate pour les livres I et VI est plus tardive que celle de Michel d'Éphèse pour les livres V, IX et X.

Quoi qu'il en soit du problème chronologique (qui est examiné de manière détaillée dans la monographie consacrée à Michel d'Éphèse), il faut signaler que le commentaire eustratien aux Seconds Analytiques, livre II, est extrêmement développé (ceux sur l'Éthique à Nicomaque, livres I et VI, le sont beaucoup moins). En effet, Eustrate a pris plusieurs initiatives touchant la forme du commentaire, notamment par rapport à celle que Michel d'Éphèse avait pratiquée dans son œuvre. La comparaison des deux œuvres exégétiques fait apparaître que, si les ouvrages de Michel étaient encore des « commentaires à scholies », en revanche, l'exégèse d'Eustrate aux Seconds Analytiques, livre II, correspondait à un commentaire à part entière, se caractérisant par une véritable continuité de l'acte exégétique; cette continuité était obtenue, de manière complète, unie et parachevée, pour l'ensemble du commentaire, mais elle était aussi pratiquée au sein de ses parties constitutives. En effet, elle était assurée grâce à un certain nombre d'initiatives qu'Eustrate semble avoir prises et qui étaient destinées à tisser un système de liens solides au sein du commentaire; parmi ces initiatives, nous nous contenterons de mentionner les suivantes.

En effet, Eustrate a dû réaliser que la lecture continue des unités exégétiques autonomes ne permettait pas d'avoir une vue précise et, à la fois, générale sur l'ensemble de l'œuvre d'Aristote examinée pas plus que sur les sections thématiques que celle-ci comportait. L'emploi de prooimia de type néoplatonicien particulièrement développés, visait précisément à doter ses commentaires d'un aperçu détaillé sur les œuvres interprétées. Quant aux différentes sections thématiques qu'il avait définies, Eustrate mettait en tête de chacune d'elles un développement général, une sorte d' " aperçu introductif », qui avait une fonction similaire à celle du prooimion général, mais à une échelle beaucoup plus réduite, étant donné qu'il ne portait que sur une seule section thématique. En ce qui concerne les unités exégétiques libres (sur ce concept, voir la définition que nous avons fournie supra), Eustrate les donnait tout de suite après les aperçus en question. Cette manière de procéder avait très probablement été influencée par la division de l'écrit exégétique néoplatonicien en théôria et praxeis. C'est ainsi que le commentaire eustratien a pu s'organiser autour des pôles thématiques fixes, dont chacun a été développé grâce, d'une part, aux aperçus introductifs et, d'autre part, aux unités exégétiques libres. 
Parmi les moyens déjà utilisés par Michel d'Éphèse, Eustrate a considérablement développé l'usage des transitions, en conférant au commentaire une forte cohésion interne du point de vue de sens; ainsi, la continuité de l'acte exégétique ne devait plus se concevoir uniquement vis-à-vis du texte d'Aristote commenté, mais, aussi, par rapport à la structure du commentaire que l'on composait. C'est dire que le commentaire a pu acquérir une autonomie textuelle et, en cessant d'être un texte susceptible de se « dilater » ou de « se réduire » de manière incontrôlable suivant les ajouts effectués ou les passages enlevés, il correspondait uniquement à la forme finale que son auteur avait voulu lui donner. Au lieu d'être, comme l'avait pensé Michel d'Éphèse, un ensemble comportant aussi des unités exégétiques originales (autrement dit des unités qui avaient été pensées et mises par écrit par l'auteur lui-même et qui, par conséquent, n'étaient plus empruntées de manière exclusive aux auteurs anciens), le commentaire est devenu, aux mains d'Eustrate, un ensemble original dans ses parties, mais, aussi, dans sa conception générale.

e. D'Eustrate de Nicée à Théodore Prodrome. — Si les exégèses composées par Eustrate marquent la naissance, à Byzance, du véritable commentaire dit «perpétuel », elles marquent aussi le point culminant de ce type d'écrit, qui ne semble pas avoir été dépassé jusqu'à la chute de Constantinople aux mains des Croisés en 1204. En effet, les modifications formelles adoptées par Eustrate (dont celles qui ont été mentionnées plus haut) se remarquent aussi dans le commentaire de Théodore Prodrome (XII ${ }^{\mathrm{e}}$ siècle) aux Seconds Analytiques, livre $\mathrm{II}^{1}$.

Plus précisément, même si ce commentaire semble avoir subi l'influence de celui d'Eustrate, il ne présente toutefois pas le même degré d'organisation et il ne se caractérise pas non plus par la même longueur dans les développements. Plus précisément, même si Théodore Prodrome s'est montré, lui aussi, soucieux à l'égard des transitions textuelles, il a opté pour un mode opératoire quelque peu différent : les Aperçus introductifs qu'il a présentés en tête des unités thématiques sont moins longs et, en règle générale, il a préféré donner plus d'ampleur aux développements qui leur succèdent, au sein desquels il a intégré, tout comme Eustrate, les unités exégétiques autonomes. De plus, Théodore Prodrome a développé davantage l'utilisation des grandes systématisations interprétatives et cette manière de procéder caractérise nettement son commentaire ${ }^{2}$.

1. Édition effectuée par nos soins : Le commentaire de Théodore Prodrome au second livre des Analytiques Postérieurs d'Aristote : texte (editio princeps et tradition manuscrite) et étude du commentaire de Prodrome (thèse de doctorat, université Paris IV-Sorbonne, 1992). Depuis, nous avons consacré plusieurs publications à ce commentaire et, de manière plus générale, à l'œuvre exégétique de Théodore Prodrome; en dernier lieu, voir le bref aperçu sur l'œuvre philosophique de cet auteur dans notre contribution : « Jean Chortasménos, Théodore Prodrome et le Recueil de "définitions" consacrées aux Seconds Analytiques, livre II. Contribution à l'étude de la tradition exégétique des Analytiques à Byzance ", volume d'hommage à D. Georgoudis, Athènes, 2007, p. 43-70, et, en particulier, p. 46-47; on peut aussi consulter les développements que nous avons consacrés à ce sujet dans le dernier volume du Dictionnaire des philosophes antiques (sous presse).

2. Ces lieux communs sont étudiés dans notre édition du commentaire de Théodore Prodrome (t. II), et, également, dans notre contribution aux Actes du congrès international pour le VIII centenaire d'Averroès, Cordoue, 9-11 décembre 1998 (sous presse). 
Ainsi, étant donné cette conception du commentaire, ce dernier se présente désormais surtout comme une succession de développements exégétiques, dont l'organisation devient possible grâce à l'emploi systématique des lieux communs qui les soutiennent.

\section{Histoire de la culture et de l'enseignement à Byzance et dans l'après- Byzance : les disciplines du trivium et du quadrivium (Arts libéraux) : corpus et manuels (suite); terminologie et lexique de l'enseignement}

Dans cette section de notre séminaire, il a tout d'abord été question du Corpus ephesianum, autrement dit du corpus d'exégèses composées par Michel d'Éphèse (voir supra, I). Plus précisément, il a été question des exégèses que ce corpus devait englober à l'origine, de la forme sous laquelle elles devaient se présenter et, notamment, des problèmes d'attribution suscités par certaines d'entre elles. En effet, ce corpus est certes de contenu philosophique, mais, étant donné son importance et, aussi, le fait qu'il avait très probablement été conçu pour constituer un corpus exégétique complet des œuvres d'Aristote (ce qui était pratiquement passé inaperçu jusqu'à présent), nous avons jugé utile de nous pencher sur sa composition. À ces raisons s'ajoutait le fait que, dans l'enseignement, la philosophie couronnait les sciences du Quadrivium (qui étaient, à leur tour, précédées des disciplines du Trivium), et que, à ce titre, le corpus en question semble avoir été « complété », lorsqu'il a été en partie reconstitué sous les Paléologues (voir supra) avec des œuvres relevant des disciplines littéraires et mathématiques.

Après avoir passé en revue ce corpus, nous avons abordé la question de la terminologie de l'enseignement à Byzance. Cette présentation est effectuée dans le cadre de l'élaboration d'un lexique de l'enseignement et ce travail repose sur le dépouillement des textes directement ou indirectement liés à l'enseignement. Nous avons commencé par présenter les différentes catégories permettant de regrouper les termes en question, puis nous avons entamé l'étude des premiers textes. Cet exposé est appelé à se prolonger l'année prochaine (2008-2009). 